\title{
T-cell responses in domestic pigs and wild boar upon infection with the moderately virulent African swine fever virus strain "Estonia2014"
}

\author{
Alexander Schäfer ${ }^{1}$, Laura Zani ${ }^{1}$, Jutta Pikalo ${ }^{1}$, Jane Hühr ${ }^{1}$, Julia Sehl ${ }^{1}$, Thomas \\ Mettenleiter $^{1}$, Angele Breithaupt ${ }^{1}$, Sandra Blome ${ }^{1}$, and Ulrike Blohm ${ }^{1}$ \\ ${ }^{1}$ Friedrich-Loeffler-Institute Federal Research Institute for Animal Health
}

September 11, 2020

\begin{abstract}
Infection with African swine fever virus (ASFV) causes a highly lethal hemorrhagic disease in domestic and Eurasian wild pigs. Thus, it is a major threat to pig populations worldwide and a cause of substantial economic losses. Recently, less virulent ASFV strains emerged naturally, which showed higher experimental virulence in wild boar than in domestic pigs. The reason for this difference in disease progression and outcome is unclear but likely involves different immunological responses. Unfortunately, besides the importance of CD $8 \alpha+$ lymphocytes, little is known about the immune responses against ASFV in suids. Against this background, we used a multicolor flow cytometry platform to investigate the T-cell responses in wild boar and domestic pigs after infection with the moderately virulent ASFV strain "Estonia2014" in two independent trials. CD4-/CD8 $\alpha+$ and $\mathrm{CD} 4+/ \mathrm{CD} 8 \alpha+\alpha \beta \mathrm{T}$-cell frequencies increased in both subspecies in various tissues, but CD8 $\alpha+\gamma \delta \mathrm{T}$ cells differentiated and responded in wild boar only. Proliferation in CD $8 \alpha+\mathrm{T}$ cells was found 10 days post infectionem only. Frequencies of T-bet $+\mathrm{T}$ cells increased in wild boar but not in domestic pigs. Of note, we found a considerable loss of perforin expression in cytotoxic $\mathrm{T}$ cells, 5 and $7 \mathrm{dpi}$. Both subspecies established a regulatory T-cell response $10 \mathrm{dpi}$. In domestic pigs, we show increasing levels of ICOS + and CD $8 \alpha+$ invariant Natural Killer T cells. These disparities in T-cell responses might explain some of the differences in disease progression in wild boar and domestic pigs and should pave the way for future studies.
\end{abstract}

T-cell responses in domestic pigs and wild boar upon infection with the moderately virulent African swine fever virus strain "Estonia2014"

Running title: T-cell responses in ASFV-Estonia infected pigs

Alexander Schäfer, Laura Zani, Jutta Pikalo, Jane Hühr, Julia Sehl, Thomas C. Mettenleiter, Angele Breithaupt, Sandra Blome, Ulrike Blohm ${ }^{\S}$

All: Friedrich-Loeffler-Institut, Greifswald - Insel Riems, Germany

ORCIDs:

Alexander Schäfer, 0000-0002-4258-0651

Laura Zani, 0000-0001-5263-8389

Jutta Pikalo, 0000-0003-3502-4240

Jane Hühr, 0000-0002-2235-9618

Julia Sehl, 0000-0002-3884-1943

Angele Breithaupt, 0000-0002-6373-5923 
Sandra Blome, 0000-0001-5465-5609

Ulrike Blohm, 0000-0003-4597-248X

${ }^{\S}$ Corresponding author:

Ulrike Blohm

Friedrich-Loeffler-Institut

Federal Research Institute for Animal Health

Südufer 10

D - 17493 Greifswald - Insel Riems

Germany

Phone: +49 383517 1543; E-mail: Ulrike.Blohm@fli.de

\section{Summary}

Infection with African swine fever virus (ASFV) causes a highly lethal hemorrhagic disease in domestic and Eurasian wild pigs. Thus, it is a major threat to pig populations worldwide and a cause of substantial economic losses. Recently, less virulent ASFV strains emerged naturally, which showed higher experimental virulence in wild boar than in domestic pigs. The reason for this difference in disease progression and outcome is unclear but likely involves different immunological responses. Unfortunately, besides the importance of CD8 $\alpha^{+}$lymphocytes, little is known about the immune responses against ASFV in suids. Against this background, we used a multicolor flow cytometry platform to investigate the T-cell responses in wild boar and domestic pigs after infection with the moderately virulent ASFV strain "Estonia2014" in two independent trials. $\mathrm{CD} 4^{-} / \mathrm{CD} 8 \alpha^{+}$and $\mathrm{CD} 4^{+} / \mathrm{CD} 8 \alpha^{+} \alpha \beta \mathrm{T}$-cell frequencies increased in both subspecies in various tissues, but $\mathrm{CD} 8 \alpha^{+} \gamma \delta \mathrm{T}$ cells differentiated and responded in wild boar only. Proliferation in CD $8 \alpha^{+} \mathrm{T}$ cells was found 10 days post infectionem only. Frequencies of T-bet ${ }^{+} \mathrm{T}$ cells increased in wild boar but not in domestic pigs. Of note, we found a considerable loss of perforin expression in cytotoxic $\mathrm{T}$ cells, 5 and $7 \mathrm{dpi}$. Both subspecies established a regulatory T-cell response $10 \mathrm{dpi}$. In domestic pigs, we show increasing levels of ICOS + and $\mathrm{CD} 8 \alpha^{+}$invariant Natural Killer T cells. These disparities in T-cell responses might explain some of the differences in disease progression in wild boar and domestic pigs and should pave the way for future studies.

Keywords : African swine fever virus, experimental infection, domestic pig, wild boar, T cell response, iNKT cells

\section{Introduction}

African Swine Fever (ASF) remains a major threat to wild and domestic pig populations . The causative agent, ASF virus (ASFV), is a large dsDNA virus and the only member in the genus Asfivirus of the Asfarviridae family . In its geographic origin in sub-Saharan Africa, ASFV is transmitted by soft ticks of the Ornithodorosgenus and circulates in other members of the Suidae, warthogs (Phacochoerus africanus ) and bushpigs (Potamochoerus larvatus). While ASFV infection causes major disease in domestic pigs and wild boar with profound manifestations and high lethality, it is clinically inapparent in its reservoir hosts, i.e., African wild suids. In European and Asian countries, the abundant wild boar populations serve as a reservoir for ASFV and present a risk for ASFV introduction into domestic pig holdings . Its near-global panzootic spread already caused death of millions of pigs in commercial and private farms and also led to major economic challenges . 
At present, little is known about the role of the host's immune response against ASFV. Once infection in a mammalian host is established, ASFV has a distinct cell tropism for myeloid cells. It replicates in monocytes and macrophages but has also been found in granulocytes. The pivotal role of lymphocytes, presumably CD $8 \alpha^{+} \mathrm{T}$ cells in particular, has been demonstrated by antibody-dependent depletion of CD8 $\alpha^{+}$cells after priming with an avirulent ASFV strain, which resulted in the loss of protection after homologous challenge .

Recently, we showed that immune responses of domestic pigs and wild boar fail to clear an infection with the highly virulent ASFV strain "Armenia08", although for different reasons. Wild boar and domestic pigs showed comparable clinical signs with substantial fatalities within the first 8 days post infectionem (dpi). However, we found activated $\mathrm{T}$ cells and signs of a cytotoxic response only in wild boar but not in domestic pigs. Moreover, T-cell proliferation was impaired in domestic pigs, while $\mathrm{CD} 4^{+} / \mathrm{CD} 8 \alpha^{+} \mathrm{T}$ cells in wild boar showed considerable proliferation. Of note, we detected a significant loss of perforin expression in both subspecies 5 dpi in CD8 $\alpha^{+}$T cells. Still, neither response was beneficial for the final disease outcome .

In the present study, we used a similar approach and investigated the immune responses of domestic pigs and wild boar after infection with the moderately virulent ASFV strain, "Estonia2014". ASFV "Estonia2014" has been shown to cause fatal disease in wild boar whereas different domestic pigs survived the experimental infection. A comparative approach with a focus on T-cell responses was used in this study to find a possible explanation for this phenomenon. Moreover, with these data we are able to compare immune responses against moderately virulent ASFV with those against highly virulent ASFV recently published .

\section{Material and Methods}

\subsection{Experimental design}

Two independent animal trials were included in this study. Domestic pigs (Sus scrofa domesticus ) were obtained from a commercial pig farm and wild boar (Sus scrofa scrofa) were provided by wildlife parks in Mecklenburg-Western Pomerania. For the infection experiments, domestic pigs and wild boar were transferred into the high containment facilities of the Friedrich-Loeffler-Institut (L3+) and were left for acclimatization for a week.

In both studies, domestic pigs and wild boar were oro-nasally inoculated with $2 \mathrm{ml}$ cell culture supernatant containing $10^{5.25}$ haemadsorbing units (HAU)/ml of ASFV "Estonia2014". A clinical score was assessed daily, based on a previously described scoring system . Rectal temperatures were measured each day in domestic pigs and at autopsy in wild boar. 6 domestic pigs and 7 wild boar were left untreated and served as controls. In trial 1, 12 German landrace pigs, 3 months of age, and 12 wild boar, 1-2 years of age, were used. 4 animals of each group were randomly chosen for autopsy 5 and 7 dpi. For trial 2, 11 German landrace pigs, 3 months of age, and 12 wild boar, 1-2 years of age, were used. Autopsies were done 4, 7, and 10 dpi with 3 animals of each group (see table 1). Results from control animals and animals investigated $7 \mathrm{dpi}$, respectively, were grouped for all applicable analyses. For scheduled autopsies or when animals reached the humane endpoint, animals were narcotized with tiletamine/zolazepam (Zoletil @), Virbac) and xylazine (Rompun ß 2\%, Bayer HealthCare) and then euthanized by intracardial injection of pentobarbital (Release, Wirtschaftsgenossenschaft deutscher Tierärzte) or exanguination. For analysis of the immune response, blood, lung, spleen, gastro-hepatic lymph node (ghLN, Lymphonodi hepatici or gastrici), as well as liver were collected.

\subsection{Virus detection}

Hemadsorption test (HAT) was performed for virus back-titration by endpoint titration on macrophages derived from peripheral blood monocytic cells of healthy donor pigs as previously described . 
Viral genome was detected in blood and tissues by real-time PCR (qPCR) using routine assays as previously described .

\subsection{Preparation of single cell suspensions}

Single cell suspensions were prepared as described previously . Briefly, spleen and ghLN were mechanically disrupted with a steel strainer. Liver sections were cleared of peripheral blood by perfusion with ice-cold PBSEDTA before further cell extraction. Samples from lungs and perfused liver were then digested (collagenase IV $\left(2 \mathrm{mg} / \mathrm{ml}\right.$; Biochrom), DNase I $(0.1 \mathrm{mg} / \mathrm{ml}$; Sigma-Aldrich) $)$ for $1 \mathrm{~h}$ at $37^{\circ} \mathrm{C}$ in serum-free cell culture media (1:1 Ham's F12/IMDM). Tissue residuals were removed by short centrifugation. The cells were washed with serum-supplemented cell culture media ( $10 \%$ fetal calf serum) and used for flow cytometry.

\subsection{Flow cytometry}

At indicated time points, whole blood and single cell suspensions of spleen, ghLN, lung, and liver were stained for flow cytometric analyses. $50 \mu \mathrm{l}$ whole blood and $50 \mu \mathrm{l}$ single cell suspensions (approx. $1 \times 10^{6}$ leukocytes) were used for staining. To identify iNKT cells, whole blood was incubated with PBS57-loaded murine CD1d (mCD1d) tetramers at room temperature for $30 \mathrm{~min}$ in the dark as described previously. All further incubation steps with monoclonal antibodies (mAbs) targeting extracellular antigens were carried out for $15 \mathrm{~min}$ at $4^{\circ} \mathrm{C}$ in the dark. Between each antibody staining, a washing step was performed. Before intracellular labeling, erythrocytes in blood samples were lysed with red blood cell lysis buffer (1.55 M $\mathrm{NH}_{4} \mathrm{Cl}, 100 \mathrm{mM} \mathrm{KHCO} 3,12.7 \mathrm{mM} \mathrm{Na}{ }_{4} \mathrm{EDTA}, \mathrm{pH}$ 7.4, in Aqua destillata ). Subsequently, samples were fixed and permeabilized with the True-Nuclear Transcription Factor Buffer Set (Biolegend, USA) according to the manufacturer's instructions. All incubation steps for intracellular staining were carried out for 30 min at $4^{\circ} \mathrm{C}$ in the dark. Antibodies and conjugates used for flow cytometry are shown in table 2. The mCD1d tetramer was obtained from the NIH Tetramer Core Facility.

Dead cells were excluded by FSC/SSC characteristics and using Zombie Aqua (Biolegend, USA). Single cells were identified by consecutive FSC-W vs. FSC-H and SSC-W vs. SSC-H gating. Live, single lymphocytes were further divided into $\mathrm{CD}^{+} / \gamma \delta \mathrm{T}$ cell receptor $(\mathrm{TCR})^{-}\left(\alpha \beta \mathrm{T}\right.$ cells), $\mathrm{CD}^{+} / \gamma \delta \mathrm{TCR}^{+}(\gamma \delta \mathrm{T}$ cells), and $\mathrm{CD}^{+} / \mathrm{mCD} 1 \mathrm{~d}$ tetramer ${ }^{+}$(iNKT cells). Further subpopulations were gated according to the markers described in the figures and text. At least $1 \times 10^{4}$ single, live $\alpha \beta \mathrm{T}$ cells were recorded. CD4 was not detectable in one of the control wild boar in trial 2, presumably because of a polymorphism in the CD4 alleles . $\mathrm{CD}^{+}$and $\mathrm{CD} 4^{+} / \mathrm{CD} 8 \alpha^{+}$cells from this animal were therefore not included in the analyses.

Flow Cytometer BD FACS Canto II with FACS DIVA Software (BD Bioscience, San Jos, CA) and FlowJo ${ }^{\text {TM }}$ V10 for Windows (Becton, Dickinson and Company; 2019) were used for all analyses.

\subsection{Statistical analysis}

Statistical analyses and graph creation were done using GraphPad Prism8 (Graphpad Software Inc., USA). Normality was verified using Shapiro-Wilk test. To investigate statistically significant differences between infected and uninfected animals, ordinary one-way ANOVA with Dunnett's correction for multiple comparisons was used. Differences between both swine subspecies were not tested because of differences at the baseline level. Data obtained in trial 1 is indicated by circles $\left(^{*}\right)$, data obtained in trial 2 is indicated by squares (). Each dot represents one animal with a bar indicating mean. Statistical significance was defined as $\mathrm{p}<0.05$ and was indicated with an asterisk $\left(^{*}\right)$. 


\section{Results}

\subsection{Clinical course}

Back-titration of the virus suspension used for inoculation verified the administered titer of $1 \times 10^{5.25}$ $\mathrm{HAU} / \mathrm{ml}$ per pig in both trials. All tissue samples from inoculated domestic pigs and wild boar were positive for ASFV genome in qPCR and gross pathology was comparable between trial 1 and 2 (data not shown). All animals developed signs of disease starting 4 dpi. Domestic pigs developed moderate disease but recovered until 10 dpi (maximum clinical score 5), whereas wild boar showed signs of moderate to severe disease until the end of the study (maximum clinical score 10.5) and thus, confirmed higher virulence of ASFV "Estonia2014" in wild boar. Details regarding viral load, histopathology, and analysis by electron microscopy of trial 2 can be found in a parallel publication .

Briefly, macroscopic pathology, ASFV antigen-positive myeloid cells investigated by flow cytometry, and viral loads in the investigated tissues showed comparable results in wild boar and domestic pigs. In contrast, higher levels of ASFV antigen were found in wild boar by immunohistochemistry.

\subsection{Altered T-cell subset frequencies in lymphoid tissue}

$\mathrm{CD}^{+} \mathrm{T}$ cells were subdivided into $\alpha \beta \mathrm{T}$ cells $\left(\mathrm{CD}^{+} / \gamma \delta \mathrm{TCR}^{-}\right)$and $\gamma \delta \mathrm{T}$ cells $\left(\mathrm{CD}^{+} / \gamma \delta \mathrm{TCR}^{-}\right) . \alpha \beta \mathrm{T}$ cells in domestic pigs showed decreases 5 dpi in blood, spleen, lung, and liver with corresponding increased $\gamma \delta$ T-cell frequencies (Figure 1A, B). The T-cell frequencies in domestic pigs returned to control levels 10 dpi, except for $\alpha \beta$ T-cell increases in the liver (Figure 1A). In wild boar, we detected temporarily increased $\alpha \beta$ T-cell frequencies in blood and spleen $4 \mathrm{dpi}$ but no other changes to baseline level (Figure 1A) and largely unaffected $\gamma \delta$ T-cell frequencies over the study period, except for corresponding decreases in $\gamma \delta$ Tcell frequencies in blood and spleen (Figure 1B). No changes were detected in the gastro-hepatic lymph nodes (ghLN, Lymphonodi hepatici or gastrici) of both subspecies.

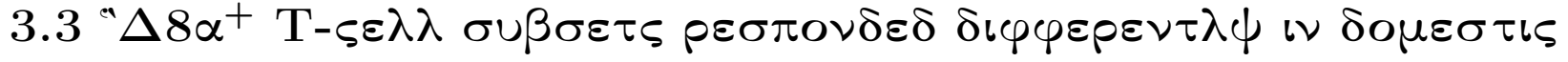 $\pi i \gamma s \alpha \nu \delta \omega \iota \lambda \delta \beta o \alpha \rho$}

CD $8 \alpha^{+}$lymphocytes are known mediators of immunity during ASFV infection. Being the largest group of $\mathrm{CD} 8 \alpha^{+}$lymphocytes, we investigated $\mathrm{CD} 8 \alpha^{+} \mathrm{T}$-cell frequencies among $\alpha \beta \mathrm{T}$ cells. Frequencies of $\mathrm{CD} 4^{-} / \mathrm{CD} 8 \alpha^{+} \alpha \beta \mathrm{T}$ cells increased in blood, spleen, lungs, and liver of domestic pigs (Figure 2A). The increase was most prominent in spleen, lung, and liver of infected animals and was significant 5 or 7 dpi until the end of the trial. In the blood, we detected increased frequencies of $\operatorname{CD} 8 \alpha^{+} \alpha \beta$ T cells 10 dpi only. $\mathrm{CD} 4^{-} / \mathrm{CD} 8 \alpha^{+} \alpha \beta \mathrm{T}$ cells in wild boar demonstrated a comparable course, except for the lung. There were no frequency alterations of $\mathrm{CD} 4^{-} / \mathrm{CD} 8 \alpha^{+} \alpha \beta \mathrm{T}$ cells in ghLN of both subspecies (Figure $2 \mathrm{~A}$ ).

Frequencies of $\mathrm{CD} 4^{+} / \mathrm{CD} 8 \alpha^{+}$double positive (DP) $\alpha \beta \mathrm{T}$ cells showed a similar but less pronounced pattern (Figure 2B). Domestic pigs had elevated levels of DP T cells 5 and 7 dpi in spleen and liver, correlating with increased levels of $\mathrm{CD}^{-} / \mathrm{CD} 8 \alpha^{+} \alpha \beta \mathrm{T}$ cells in the same samples (Figure 2A, B). We also found increased frequencies of DP $\alpha \beta$ T cells in the lungs and ghLN 7 dpi. There were no significant changes in the blood. In wild boar, we found increased DP $\alpha \beta$ T-cell frequencies 5 dpi in the liver, 5 and 7 dpi in the lungs and 7 dpi in the blood (Figure 2B). Alterations in both subspecies were only temporary, as DP T cell frequencies returned to baseline levels in all animals $10 \mathrm{dpi}$.

$\gamma \delta \mathrm{T}$ cells are also known to express CD $8 \alpha$ upon activation and differentiation into CD2 ${ }^{+} / \mathrm{CD} 8 \alpha^{+}$effector cells. We did not detect any changes in effector $\gamma \delta$ T-cell frequencies in the investigated tissues in domestic pigs (Figure 3). In contrast, effector $\gamma \delta$ T-cell frequencies in wild boar increased in spleen 5 and 7 dpi, and 
$7 \mathrm{dpi}$ in lung. Most pronounced and persistent increases were found in the liver of infected animals 4 to 7 dpi (Figure 3). Comparable to DP $\alpha \beta$ T cells, the changes were not permanent and effector $\gamma \delta$ T-cell levels returned to control levels $10 \mathrm{dpi}$.

We also analyzed Ki-67 expression as a marker for proliferation in the second trial. We found pronounced proliferation of CD8 $\alpha \alpha^{+}$and CD8 $\alpha \beta^{+}$T cells in domestic pigs as well as in wild boar 10 dpi (Figure 4A, B). Proliferating cells were primarily found $10 \mathrm{dpi}$, but elevated frequencies of Ki-67 ${ }^{+}$CD8 $\alpha \alpha^{+}$and CD8 $\alpha \beta^{+} \mathrm{T}$ cells were also found $7 \mathrm{dpi}$ in the spleen of domestic pigs. Regarding CD4 ${ }^{+} \alpha \beta \mathrm{T}$ cells, we found significantly increased frequencies of proliferating $\mathrm{CD} 4^{+} / \mathrm{CD} 8 \alpha^{-} \mathrm{T}$ cells only in the spleen of both suid species $7 \mathrm{dpi}$. Moreover, DP T cells in domestic pigs proliferated significantly 7 and $10 \mathrm{dpi}$, again only in spleen (Supplemental figure 1). In contrast, we did not find $\mathrm{Ki}-67^{+}$, proliferating $\gamma \delta \mathrm{T}$ cells in domestic pigs or wild boar (data not shown).

\subsection{Perforin levels were decreased in cytotoxic $\mathrm{T}$ cells}

To investigate the ability of cytotoxic lymphocytes to clear virus-infected cells, we analyzed the expression of one of the major cytotoxic effector molecules, perforin, in cytotoxic lymphocyte populations, CD8 $\alpha \alpha^{+}$and CD $8 \alpha \beta^{+} \alpha \beta$ T cells, and effector $\gamma \delta \mathrm{T}$ cells (Figure 5).

In contrast to all other investigated tissues, perforin expression in CD8 $\alpha \alpha^{+} \mathrm{T}$ cells was increased 7 dpi in blood and spleen of domestic pigs (Figure 5A, B). Strikingly, it was reduced 4 dpi and virtually undetectable 5 dpi in the liver and ghLN of domestic pigs (Figure 5C, D). In wild boar, perforin expression decreased 5 dpi in the blood, but was elevated above control levels 7 and 10 dpi in blood, spleen, liver, and ghLN (Figure $5 \mathrm{~A}-\mathrm{D})$.

CD8 $\alpha \beta^{+} \mathrm{T}$ cells showed a similar but more pronounced course with a significantly decreased expression of perforin 4 and 5 dpi in domestic pigs as well as wild boar in all investigated tissues (Figure 5A-D). Compared to the perforin expression in uninfected controls, the perforin loss in CD8 $\alpha \beta^{+} \mathrm{T}$ cells was even more distinct than in CD8 $\alpha \alpha^{+} \mathrm{T}$ cells. Perforin expression was back to control levels 7 dpi in all animals. In domestic pigs, another significant decrease of perforin occurred $10 \mathrm{dpi}$. This second reduction was less pronounced in wild boar.

CD $8 \alpha^{+}$effector $\gamma \delta \mathrm{T}$ cells showed a perforin expression comparable to CD $8 \alpha \alpha^{+} \mathrm{T}$ cells. In domestic pigs, the perforin expression was increased 7 dpi in the blood (Figure 5A) but remained virtually unchanged in the spleen (Figure 5B). Wild boar showed a reduced perforin expression $5 \mathrm{dpi}$ in blood and spleen. However, this effect was less pronounced as for the other T-cell populations and returned to control levels 7 and 10 dpi (Figure 5A, B). In the liver and ghLN, we detected slight perforin decreases in ghLN 10 dpi but no other significant expression changes in wild boar (Figure 5C, D). In contrast, perforin expression in domestic pigs showed a significant decrease 5 dpi in the liver, and 4 and 5 dpi in ghLN. After the cells regained their perforin expression levels $7 \mathrm{dpi}$, there was another loss $10 \mathrm{dpi}$ (Figure 5C, D).

\subsection{T-bet-dependent T-cell activation was found in wild boar but not domestic pigs}

Expression of the T-box transcription factor TBX21 (T-bet) in $\alpha \beta$ and $\gamma \delta \mathrm{T}$ cells was investigated as a marker for activation. Domestic pigs had relatively high levels of T-bet ${ }^{+}$cells even before the infection and thus, there was no further activation measurable over the course of the study. In blood and spleen, T-bet expression was decreased below baseline levels (Supplemental figure 2). In contrast, we detected increased frequencies of T-bet ${ }^{+} \mathrm{CD} 4^{-} / \mathrm{CD} 8 \alpha^{+} \alpha \beta \mathrm{T}$ cells in spleen, lung, and liver of infected wild boar 10 dpi (Figure 6). Moreover, we found heightened proportions of T-bet ${ }^{+} \mathrm{CD} 4^{+} / \mathrm{CD} 8 \alpha^{-} \alpha \beta \mathrm{T}$ cells $10 \mathrm{dpi}$ and of all differentiation states of $\gamma \delta \mathrm{T}$ cells 7 and $10 \mathrm{dpi}$ in the lung of infected wild boar (Figure 6). 


\subsection{Regulatory $\mathrm{T}$ cells were induced in both subspecies}

FoxP $^{+}$cells among $\mathrm{CD} 4^{+} / \mathrm{CD} 8 \alpha^{-}$and $\mathrm{CD} 4^{+} / \mathrm{CD} 8 \alpha^{+} \mathrm{T}$ cells were analyzed to identify regulatory $\mathrm{T}$-cell responses. In spleen, lung, and ghLN, Treg frequencies increased significantly 7 or 10 dpi in domestic pigs and wild boar, with the highest frequencies in the lungs of both subspecies (Figure 7). Interestingly, although Tregs were induced earlier in domestic pigs, Treg frequencies reached higher levels in wild boar. Of note, there were more DP Tregs than $\mathrm{CD} 4^{+} / \mathrm{CD} 8 \alpha^{-}$Tregs in both subspecies (Figure 7 ).

\section{7 iNKT cells in domestic pigs were activated}

During infection with highly virulent ASFV "Armenia08", we described iNKT-cell frequency fluctuations in disease-affected tissues. In trial 2 of this study, we investigated iNKT-cell frequency and expression of activation markers (CD25, ICOS, and MHC II) and maturation markers (CD4 and CD8 $\alpha$ ) on iNKT cells of domestic pigs according to previous findings. We did not find changes in iNKT-cell frequency or CD25 expression (Supplemental figure 3). ICOS was upregulated on iNKT cells in blood, spleen, and ghLN 7 and 10 dpi (Figure 8A). Moreover, frequencies of $\mathrm{CD} 4^{-} / \mathrm{CD} 8 \alpha^{+}$and $\mathrm{CD} 4^{+} / \mathrm{CD} 8 \alpha^{+}$iNKT cells increased in the blood of infected animals and to a lesser extent also in the spleen, lung, and liver (Figure 8A, B). Of note, MHC II was downregulated on iNKT cells in the blood 4 dpi and in the liver 10 dpi (Figure 8D). We were not able to identify an iNKT-cell population in wild boar and thus could not investigate iNKT-cell responses in those animals.

\section{Discussion}

The porcine T-cell response against ASFV infection is largely not understood. Moreover, knowledge about the responses of susceptibleSuidae subspecies outside of Africa, domestic pigs (Sus scrofa domesticus ) and wild boar (Sus scrofa scrofa), is scarce. Similar to our first approach to analyze these differences upon infection with the highly virulent ASFV strain "Armenia08", we used a multicolor flow cytometry platform to investigate T-cell responses in domestic pigs and wild boar after infection with the moderately virulent ASFV isolate, "Estonia2014".

The most fundamental characteristics of an ongoing immune response are local or systemic alterations in the composition of leukocytes in tissues affected by disease. As one of the few known parameters of protection against ASFV infection, the importance of (cytotoxic) CD $8 \alpha^{+}$lymphocytes has been demonstrated, although to a limited extent only. It has been shown that isolated porcine PBMC, afterin vivo priming with virulent ASFV, were able to specifically lyse ASFV-infected cells in vitro. This was a first indication of virus-specific cytotoxicity. However, PBMCs were not differentiated and protection was not investigated. SLA I- and CD8-dependent lysis of ASFV-infected target cells by PBMCs from ASFV-immune minipigs and specific lysis of isolated $\mathrm{CD} 8 \alpha^{+}$but not $\mathrm{CD} 4^{+} \mathrm{T}$ cells has also been shown. In another study, antibody-dependent depletion of $\mathrm{CD} 8 \alpha^{+}$cells in vivo in domestic pigs primed with the low virulent ASFV strain "OUR/T88/3" resulted in loss of protection after homologous challenge with the virulent ASFV strain "OUR/T88/1" . We could show that the $\mathrm{CD} 8 \alpha$ response in wild boar and domestic pigs during infection with moderately virulent ASFV is based primarily on increases of $\mathrm{CD} 4^{-} / \mathrm{CD} 8 \alpha^{+}$and to a lesser extent of CD4 ${ }^{+} / \mathrm{CD} 8 \alpha^{+}$(DP) T cells. Interestingly, in our previous study with the highly virulent ASFV "Armenia08", the CD8 $\alpha$ response was primarily based on DP T cells . CD $4^{+} / \mathrm{CD} 8 \alpha^{+}$DP T cells are often described to possess memory functions . ASFV-specific memory responses can be excluded during experimental ASFV infection with naïve pigs. However, ASFV-specific responses might play a role in field infections since serological evidence for previous ASFV infections was found in hunted animals. On the other hand, DP memory T cells in the spleen might be activated independent of their cognate antigen by release of IL-15 or IL-18. Porcine DP T cells are also described to exhibit effector functions, like cytotoxic responses or cytokine production . However, in contrast to $\mathrm{CD} 8 \alpha \alpha^{+}$or $\mathrm{CD} 8 \alpha \beta^{+} \alpha \beta \mathrm{T}$ cells, we only found proliferating DP $\mathrm{T}$ cells in the spleen but not in 
other tissues. Moreover, the pronounced loss of perforin in other cytotoxic T-cell populations was not found in DP T cells. This might indicate that DP T cells are orchestrators of systemic responses but do not take part in antiviral responses in disease-affected tissue during moderately virulent ASFV infection.

Pigs belong to a group of mammals with relatively high frequencies of $\gamma \delta \mathrm{T}$ cells. They can exert effector functions like cytokine production and cytotoxicity, and are even able to present antigens to other lymphocytes . The main effector population is characterized as $\mathrm{CD} 2^{+} / \mathrm{CD} 8 \alpha^{+}$. In the present study, we found pronounced increases of effector $\gamma \delta$ T-cell frequencies in spleen, lung, and liver of infected wild boar but not domestic pigs. Moreover, we detected T-bet-dependent activation of $\gamma \delta \mathrm{T}$ cells in wild boar only. This is in line with our previous findings during highly virulent ASFV infection, where wild boar were found to have a considerably stronger bias for $\gamma \delta$ T-cell responses. This indicates a profound dissimilarity in the antiviral responses of both subspecies and might give an explanation for their different disease severity and survival. Of note, this is in contrast to previous findings, where higher frequencies of circulating $\gamma \delta \mathrm{T}$ cells correlated with increased survival of infection with moderately virulent ASFV, independent of age or virus dose . There might be some explanations for this discrepancy. First, we detected increased lethality and heightened frequencies of effector $\gamma \delta \mathrm{T}$ cells in wild boar, while the aforementioned study used domestic pigs. While we were also not able to detect changes of $\gamma \delta$ T-cell frequencies in domestic pigs, this might be caused by different ASFV strains used. Finally, the numbers of survivors that showed correlations with $\gamma \delta$ T-cell levels in the study by Post et al. were relatively small. This underlines the need for in-depth research not only during ASFV infection in general but also for the differences between wild boar and domestic pigs. Moreover, since infection of professional antigen-presenting cells alter their function, it would be of interest to investigate whether $\gamma \delta \mathrm{T}$ cells take part in the antigen presentation during ASFV infection.

We found regulatory $\mathrm{T}$ cells (Tregs) in both subspecies but higher frequencies in wild boar. The role of Tregs during ASFV infection is largely unexplored. However, previous studies showed that Tregs might present a way of viral immune evasion because they were able to inhibit specifically antiviral responses . Higher percentages of Tregs in wild boar might therefore be an explanation for their higher disease burden in this study and lethality previously. In a parallel study by Sehlet al. using histopathology from tissues of trial 2 of this study, domestic pigs but not wild boar showed lymphohistiocytic interstitial pneumonia even 10 dpi . This might be a sign for prolonged pro-inflammatory responses in domestic pigs in contrast to wild boar. This is in line with our findings of higher Treg frequencies in wild boar. Moreover, this indicates that pro-inflammatory responses are able to counteract ASFV infection, as long as they are not downregulated too early.

A porcine T-cell population that is still not well understood is invariant Natural Killer T (iNKT) cells. We could previously show that iNKT-cell frequencies significantly increased in some tissues during infection with highly virulent ASFV . Although at the time we were not able to investigate effector mechanisms or surface markers on iNKT cellsex vivo, our study provided first evidence that iNKT cells participate in the antiviral response during ASFV infection. The fact, that we were unable to find changes in the general iNKT-cell frequency might be explained by the less virulent ASFV strain in this study. Nevertheless, activation of iNKT cells was shown by significantly increased frequencies of $\mathrm{ICOS}^{+}$iNKT cells. ICOS is an essential protein for iNKT-cell activation, homeostasis, and survival. Some studies correlated ICOS expression on iNKT cells with pro-inflammatory Th1 responses, while others described it as a marker of effector iNKT cells . Increased expression of CD $8 \alpha$ and CD4, as previously established markers of maturation of porcine iNKT cells, underlines these findings. Interestingly, a role for NKT cells has previously been suggested, as $\mathrm{CD}^{+} / \mathrm{CD} 4^{-} / \mathrm{CD} 8 \alpha^{+} / \mathrm{CD}^{ \pm} / \mathrm{CD} 6^{-} / \mathrm{CD} 11 b^{+} / \mathrm{CD} 16^{+}$cells expanded after in vitro stimulation of porcine PBMC with ASFV . However, even though we and others could show that the phenotype of iNKT cells differs from that finding, the significant alterations in iNKT-cell frequency in our first study and our findings in this study support the notion that iNKT cells take part in the antiviral response against ASFV.

Besides analysis of the cellular composition of leukocytes in the investigated tissues, effector functions are also pivotal to understand the underlining immune mechanisms. Perforin is one of the major lytic molecules used by cytotoxic lymphocytes to kill target cells. Instead of direct cell lysis, cytotoxic lymphocytes can 
also induce apoptosis in their target cells by death receptor-mediated pathways using Fas ligand (FasL) or TRAIL. The significant and partially complete loss of perforin 4 to 5 dpi in this study resembled the observed loss of perforin we found during infection with the highly virulent ASFV strain "Armenia08" . However, the perforin decrease was more pronounced during infection with highly virulent ASFV, especially on a systemic level, i.e. in the spleen. There are various explanations for the perforin loss observed in both studies. Perforin-mediated killing is thought to be the major pathway in the early cytotoxic response but can be switched to Fas/FasL-mediated apoptosis induction with a complete loss of perforin expression on RNA and protein level during the course of infection. An effector molecule switch might be an explanation for the observations in our study, however, this is not directly detectable because antibodies against porcine FasL are still missing. Still, there are some lines of evidence suggesting this might be the case. Expression of viral homologues of the mammalian anti-apoptotic protein Bcl-2 and also prevention of apoptosis by these viral homologues has been shown for ASFV strains . Bcl-2 is also known to preferentially inhibit perforin-mediated apoptosis but less Fas/FasL-mediated apoptosis, depending on the cellular target. A switch from perforinmediated to Fas/FasL-mediated cytotoxic responses might therefore be beneficial and protective and would be in line with the lower disease severity and heightened survival of domestic pigs. Wild boar, in contrast, had higher levels of perforin ${ }^{+}$lymphocytes on average, indicating that they might not have switched the cytotoxic pathway or at least not to the extent that domestic pigs did. Given that inhibition of perforin has been shown to protect from tissue damage during viral hepatitis, this might also be an explanation for the more severe inflammation and tissue degradation in the liver of infected wild boar. On the other hand, it cannot be excluded that we missed newly synthesized and immediately secreted perforin, which is not detected by antibody clone dG9 used in this study . However, missing detection due to immediate secretion would still hint to a strong cytotoxic response. In this case, the cytotoxic response would have been higher in domestic pigs because the perforin loss was detected earlier and more pronounced than in wild boar. Therefore, it can be hypothesized that the more pronounced and earlier response in domestic pigs was beneficial and protective at least during infection with moderately virulent ASFV. Wild boar, in contrast, might not have been able to counter the infection because of their impaired response, eventually leading to death as observed in previous studies. Which explanation holds true, switch of cytotoxic pathways or earlier and stronger cytotoxic response, has to be investigated in future studies. It still has to be kept in mind that cytotoxic responses are not only beneficial but might also cause immunopathology and thus, contribute to disease burden .

In summary, we described the first comparative analysis of immune responses of wild boar and domestic pigs during moderately virulent ASFV infection. While more severe in wild boar, domestic pigs showed signs of moderate disease and recovery of all animals. Overall, we found comparable courses of immunity in both subspecies. Both developed a heavily CD $8 \alpha^{+}$-biased response with proliferation of CD $8 \alpha \alpha^{+}$and $\mathrm{CD} 8 \alpha \beta^{+}$cells. However, although their $\alpha \beta$ T-cell responses were largely similar, wild boar developed a more pronounced effector $\gamma \delta$ T-cell response. We also found only small signs of T-bet-dependent activation predominately in lungs and liver of wild boar but none in domestic pigs. Moreover, we found a distinct loss of perforin in cytotoxic $\mathrm{T}$ cells in domestic pigs and to a lesser extent also in wild boar, similar to previous results during infection with highly virulent ASFV "Armenia08". Tregs appeared in higher levels in wild boar. Finally, we were able show the first description of functional iNKT-cell responses during ASFV infection. With this data, our study paves the way for further in-depth analyses of porcine immunity towards ASFV.

\section{Acknowledgements}

The authors thank Silke Rehbein, Cathleen Klement, and Stefanie Knöfel for excellent technical assistance in the laboratory. For exceptional care of animals and support during the trials, we thank the animal caretakers Thomas Möritz, Steffen Brenz, Harald Manthei, and Frank Klipp. Furthermore, we thank Christian Loth for outstanding autopsy assistance. We are grateful to the NIH Tetramer Core Facility for providing the mCD1d tetramer. 


\section{Ethics statement}

All applicable animal welfare regulations, including EU Directive 2010/63/EC and institutional guidelines, were taken into consideration in the present study. The animal experiments were approved by the State Office for Agriculture, Food Safety and Fishery in Mecklenburg-Western Pomerania (LALFF M-V) under reference numbers LALLF 7221.3-1.1-064/17 and LALLF 7221.3-2-011/19.

\section{Conflict of Interest}

The authors declare no conflict of interest.

\section{Author Contributions}

Conceived and designed experiments: TCM, AB, SB, UB. Acquired animal samples: LZ, JP, JS, JH, AB, SB, UB. Sample processing: AS, JP, JH. Data analysis and interpretations: AS, UB. Manuscript preparation: AS, TCM, SB, UB. All authors reviewed and approved the final version of the manuscript.

\section{Funding}

This work was funded through the Friedrich-Loeffler-Institut African Swine Fever Virus Research Network 'ASF-ResNet'.

\section{Data availability statement}

The data supporting the findings of this study are available from the corresponding author upon reasonable request.

\section{References}

\section{Tables}

Table 1 Experimental design.

\begin{tabular}{|c|c|c|c|c|c|c|c|c|c|c|c|c|}
\hline & day & 0 & 1 & 2 & 3 & 4 & 5 & 6 & 7 & 8 & 9 & 10 \\
\hline Trial & WB & Infection & & & & & 4 & & 4 & & & \\
\hline \multirow{3}{*}{$\begin{array}{l}\text { Trial } \\
2\end{array}$} & DP & & & & & & 4 & & 4 & & & \\
\hline & WB & & & & & 3 & & & 3 & & & 3 \\
\hline & DP & & & & & 3 & & & 3 & & & 3 \\
\hline
\end{tabular}

WB, wild boar; DP, domestic pigs

Table 2 Antibodies used in this study. 


\begin{tabular}{|c|c|c|c|c|c|}
\hline Marker & Clone & Isotype & Conjugate & Source & Dilution \\
\hline CD2 & MSA4 & mouse IgG2a & - & in-house & $1: 100$ \\
\hline CD3e & PPT3 & mouse IgG1 & $\mathrm{APC}$ & Southern Biotech & $1: 500$ \\
\hline CD3e & РPT3 & mouse IgG1 & $\mathrm{PE}$ & Southern Biotech & $1: 500$ \\
\hline CD4a & $74-12-4$ & mouse IgG2b & PerCp-Сy5.5 & $\mathrm{BD}$ & $1: 100$ \\
\hline CD8a & $76-2-11$ & mouse IgG2a & FITC & Southern Biotech & $1: 100$ \\
\hline CD8b & PG164A & mouse IgG2a & - & in-house & $1: 1000$ \\
\hline Foxp3 & FJK-16s & mouse IgG1 & PE-Cy7 & ebioscience & $1: 200$ \\
\hline gdTCR & PPT16 & mouse IgG2b & - & in-house & $1: 100$ \\
\hline Ki-67 & B56 & mouse IgG1 & BV421 & BD Biosciences & $1: 40$ \\
\hline Perforin & dG9 & mouse IgG2b & Alexa 647 & Biolegend & $1: 20$ \\
\hline T-bet & $4 \mathrm{~B} 10$ & mouse IgG1 & $\mathrm{APC}$ & Biolegend & $1: 500$ \\
\hline $\operatorname{IgG} 2 \mathrm{a}$ & polyclonal & Goat IgG & APC-Cy7 & Southern Biotech & $1: 250$ \\
\hline $\operatorname{IgG} 2 b$ & polyclonal & Goat IgG & $\mathrm{PE}$ & Southern Biotech & $1: 400$ \\
\hline IgG2b & polyclonal & Goat IgG & $\mathrm{PE}-\mathrm{Cy} 7$ & Southern Biotech & $1: 400$ \\
\hline
\end{tabular}

\section{Figure legends}

Figure 1 T-cell subset variations in various tissues upon moderately virulent ASFV infection. At the indicated time points, domestic pigs (orange) and wild boar (blue) were euthanized and lymphocytes of the respective tissues were isolated. $\mathrm{CD}^{+}$lymphocytes were subdivided into $\gamma \delta$ T cell receptor (TCR) ${ }^{-}$ $\alpha \beta \mathrm{T}$ cells and $\gamma \delta \mathrm{TCR}^{+} \gamma \delta \mathrm{T}$ cells. Frequency of $(\mathbf{A}) \alpha \beta$ and $(\mathbf{B}) \gamma \delta \mathrm{T}$ cells in blood, spleen, lung, liver, and ghLN. Each point represents data from a single pig while bars represent the means for the designated time points. Data from trial $1(*)$, data from trial 2()$.{ }^{*}, \mathrm{p}<0.05$. ghLN, gastro-hepatic lymph node

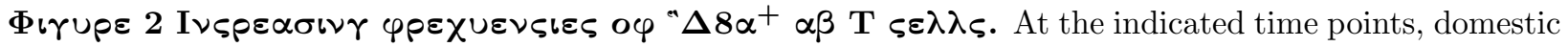
pigs (orange) and wild boar (blue) were euthanized and lymphocytes of the respective tissues were isolated. $\mathrm{CD}^{+} \alpha \beta \mathrm{T}$ cells were analyzed for CD4 and CD8 $\alpha$ expression. Frequency of (A ) CD4 ${ }^{-} / \mathrm{CD} 8 \alpha^{+} \alpha \beta \mathrm{T}$ cells and (B ) $\mathrm{CD}^{+} / \mathrm{CD} 8 \alpha^{+} \alpha \beta$ T cells in blood, spleen, lung, liver, and ghLN. Each point represents data from a single pig while bars represent the means for the designated time points. Data from trial $1\left(^{*}\right)$, data from trial 2()${ }^{*}, \mathrm{p}<0.05$. ghLN, gastro-hepatic lymph node

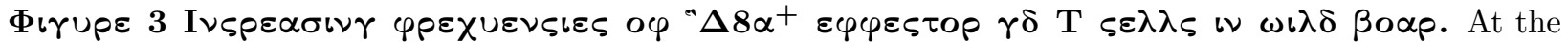
indicated time points, domestic pigs (orange) and wild boar (blue) were euthanized and lymphocytes of the respective tissues were isolated. CD3 ${ }^{+} \gamma \delta \mathrm{T}$ cells were analyzed for CD8 $\alpha$ expression. Frequency of CD8 $\alpha^{+}$ үo T cells (A ) spleen, (B ) lung, (C ) liver, and (D ) ghLN. Each point represents data from a single pig while bars represent the means for the designated time points. Data from trial $1\left(^{*}\right)$, data from trial 2() . $^{*}$ $\mathrm{p}<0.05$. ghLN, gastro-hepatic lymph node

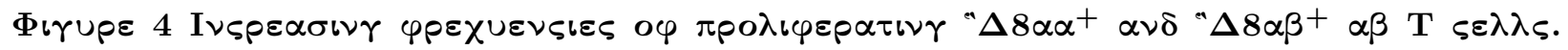
At the indicated time points, domestic pigs (orange) and wild boar (blue) were euthanized and lymphocytes of the respective tissues were isolated. $\mathrm{CD} 8 \alpha^{+} \alpha \beta \mathrm{T}$ cells were subdivided based on their expression of the CD8 $\beta$ chain. Frequency of $(\mathbf{A}) \mathrm{CD} 8 \alpha \alpha^{+}$and (B ) CD $8 \alpha \beta^{+} \mathrm{T}$ cells in blood, spleen, lung, and liver. Each point represents data from a single pig while bars represent the means for the designated time points. ${ }^{*}, \mathrm{p}$ $<0.05$.

Figure 5 Loss of perforin expression in cytotoxic $\mathbf{T}$ cells. At the indicated time points, domestic pigs (orange) and wild boar (blue) were euthanized and lymphocytes were isolated. (A ) CD8 $\alpha \alpha^{+}$and CD8 $\alpha \beta^{+} \alpha \beta$ $\mathrm{T}$ cells, and (B ) $\mathrm{CD} 2^{+} / \mathrm{CD} 8 \alpha^{+}$effector $\gamma \delta \mathrm{T}$ cells were investigated for perforin expression in the indicated tissues. Each point represents data from a single pig while bars represent the means for the designated time points. Data from trial $1(*)$, data from trial 2()${ }^{*}, \mathrm{p}<0.05$. ghLN, gastro-hepatic lymph node 
Figure 6 T-bet-dependent T-cell activation in wild boar. At the indicated time points, wild boar were euthanized and lymphocytes were isolated. (A ) $\mathrm{CD} 4^{+} / \mathrm{CD} 8 \alpha^{-}, \mathrm{CD} 4^{-} / \mathrm{CD} 8 \alpha^{+}$, and $\mathrm{CD} 4^{+} / \mathrm{CD} 8 \alpha^{+} \alpha \beta$ $\mathrm{T}$ cells (from left to right) as well as (B ) activated and effector $\gamma \delta \mathrm{T}$ cells were analyzed for their expression of T-bet. T-bet expression in domestic pigs for comparison can be found in Supplemental figure 2. Each point represents data from a single pig while bars represent the means for the designated time points. ${ }^{*}, \mathrm{p}$ $<0.05$. ghLN, gastro-hepatic lymph node

Figure 7 FoxP3 ${ }^{+}$regulatory $\mathrm{T}$ cells appeared in domestic pigs and wild boar. At the indicated time points, domestic pigs (orange) and wild boar (blue) were euthanized and lymphocytes of the respective tissues were isolated. $\mathrm{CD} 4^{+} / \mathrm{CD} 8 \alpha^{-}$or $\mathrm{CD} 4^{+} / \mathrm{CD} 8 \alpha^{+} \alpha \beta$ T cells were analyzed for their expression of FoxP3. Frequency of $(\mathbf{A}) \mathrm{FoxP}^{+} / \mathrm{CD} 4^{+} / \mathrm{CD} 8 \alpha^{-}$and $(\mathbf{B}) \mathrm{FoxP} 3^{+} / \mathrm{CD} 4^{+} / \mathrm{CD} 8 \alpha^{+} \mathrm{T}$ cells in blood, spleen, and ghLN. Each point represents data from a single pig while bars represent the means for the designated time points. *, $\mathrm{p}<0.05$. ghLN, gastro-hepatic lymph node

Figure 8 Activation and differentiation of iNKT cells in domestic pigs. At the indicated time points, domestic pigs were euthanized and lymphocytes of the respective tissues were isolated. $\mathrm{CD} 3^{+} \mathrm{mCD} 1 \mathrm{~d}$ tetramer ${ }^{+}$iNKT cells were analyzed by flow cytometry. Frequency of iNKT cells positive for (A ) ICOS, (B ) MHC II, (C ) CD $8 \alpha$, and (D ) CD4/CD $8 \alpha$. Each point represents data from a single pig while bars represent the means for the designated time points. ${ }^{*}, \mathrm{p}<0.05$. ghLN, gastro-hepatic lymph node

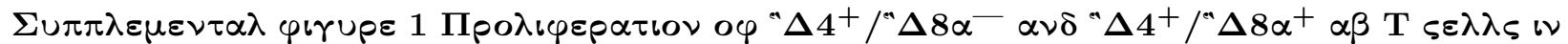
$\tau \eta \varepsilon \sigma \pi \lambda \varepsilon \varepsilon \nu$. At the indicated time points, domestic pigs (orange) and wild boar (blue) were euthanized and lymphocytes of the respective tissues were isolated. Frequency of (A) $\mathrm{CD} 4^{+} / \mathrm{CD} 8 \alpha^{-}$and $(\mathbf{B}) \mathrm{CD} 4^{+} / \mathrm{CD} 8 \alpha^{+}$ $\mathrm{T}$ cells in spleen. Each point represents data from a single pig while bars represent the means for the designated time points. ${ }^{*}, \mathrm{p}<0.05$.

Supplemental figure 2 No evidence of T-bet-dependent activation in domestic pigs. At the indicated time points, domestic pigs (orange) were euthanized and lymphocytes of the respective tissues were isolated. Frequency of T-bet ${ }^{+}$cells in (A ) blood, (B ) spleen, (C ) liver, and (D ) ghLN. Each point represents data from a single pig while bars represent the means for the designated time points. ${ }^{*}, \mathrm{p}<0.05$. ghLN, gastro-hepatic lymph node

Supplemental figure 3 Frequency and CD25 expression of iNKT cells in domestic pigs. At the indicated time points, domestic pigs were euthanized and lymphocytes of the respective tissues were isolated. $\mathrm{CD}^{+}$mCD1d tetramer ${ }^{+}$iNKT cells were analyzed by flow cytometry. Frequency of iNKT cells (A ) of $\mathrm{CD}^{+}$cells and $(\mathbf{B})$ positive for CD25. Each point represents data from a single pig while bars represent the means for the designated time points. ${ }^{*}, \mathrm{p}<0.05$. ghLN, gastro-hepatic lymph node 


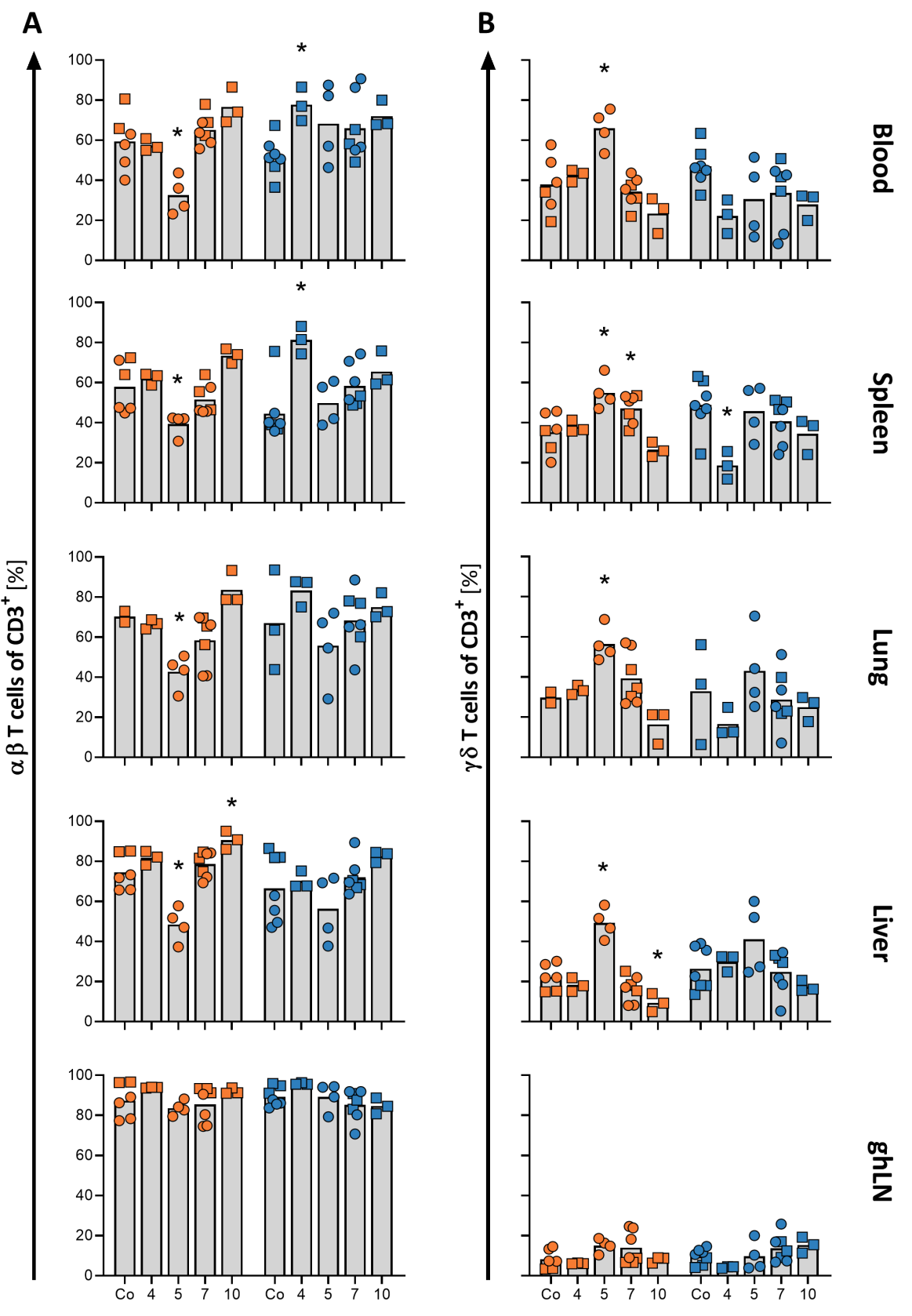



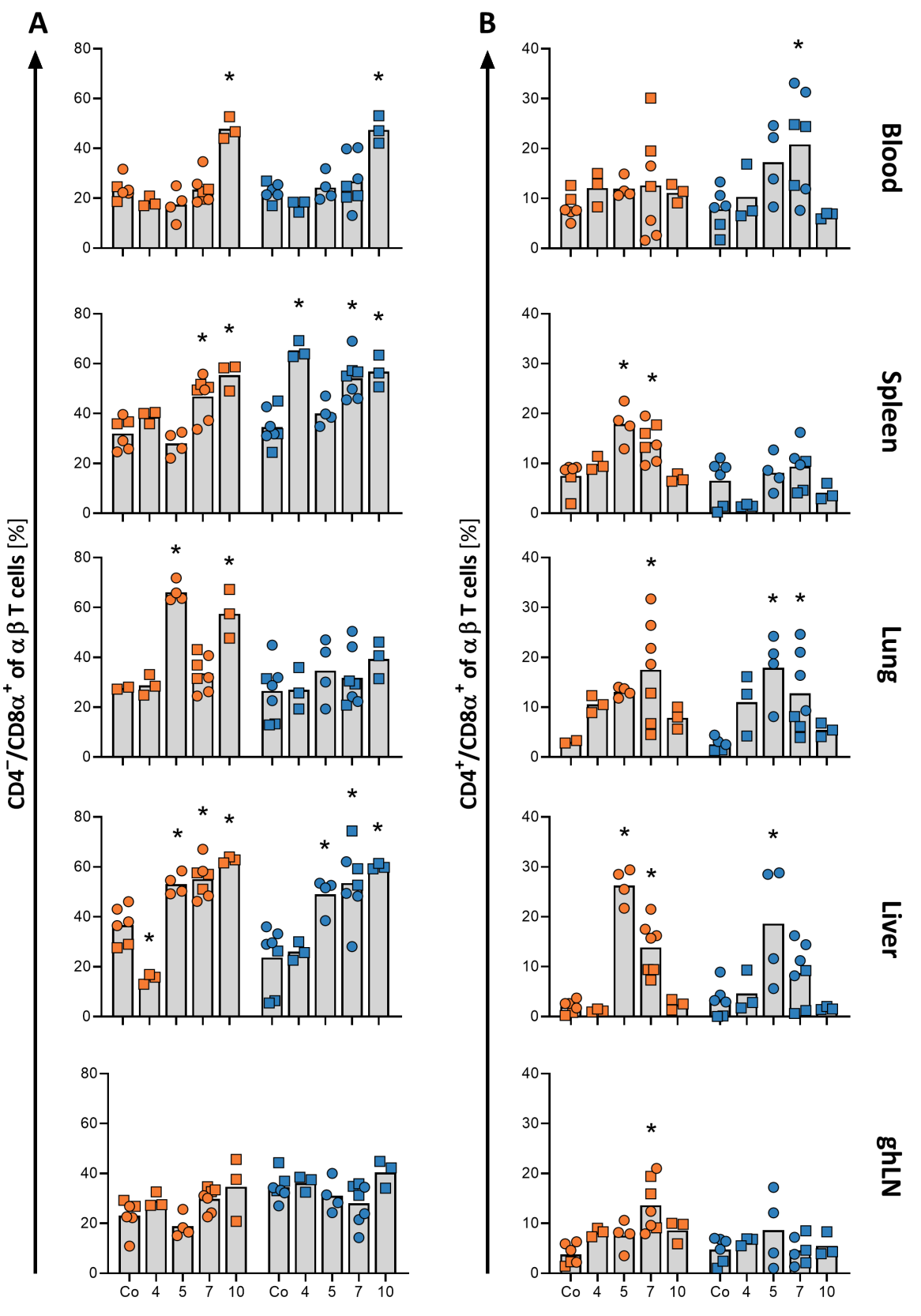


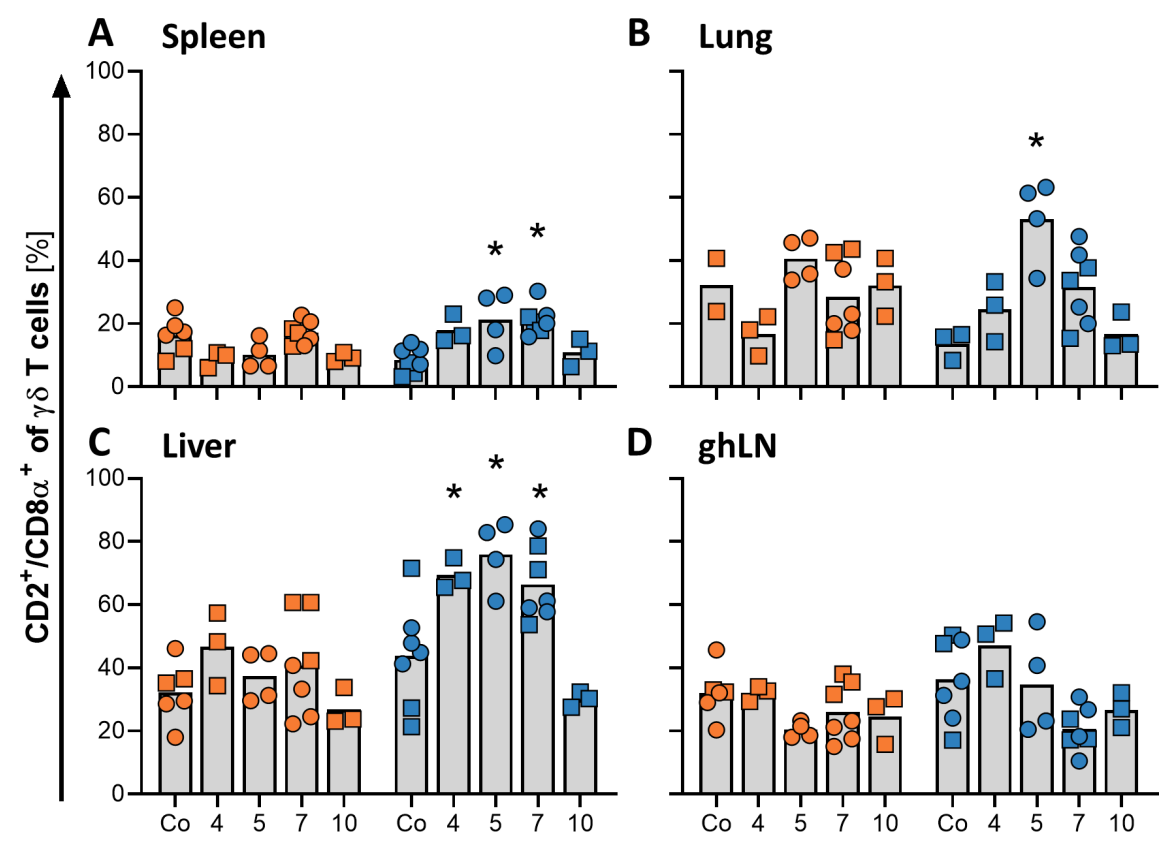


A

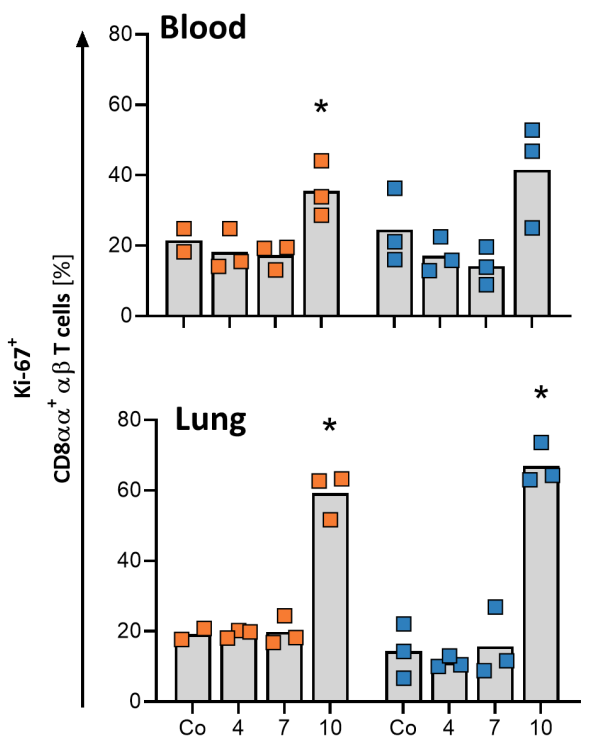

B

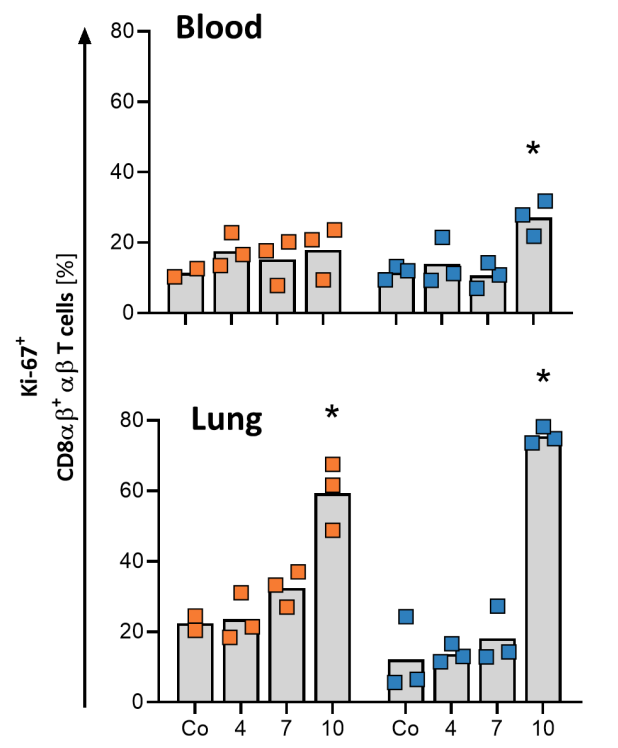

Spleen
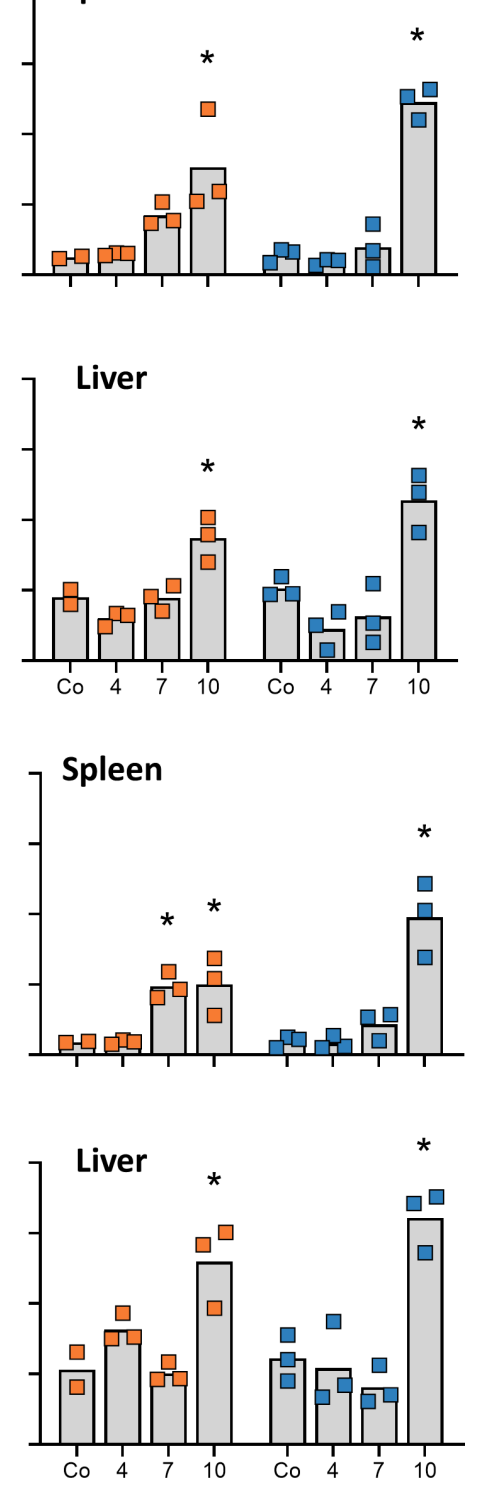
A
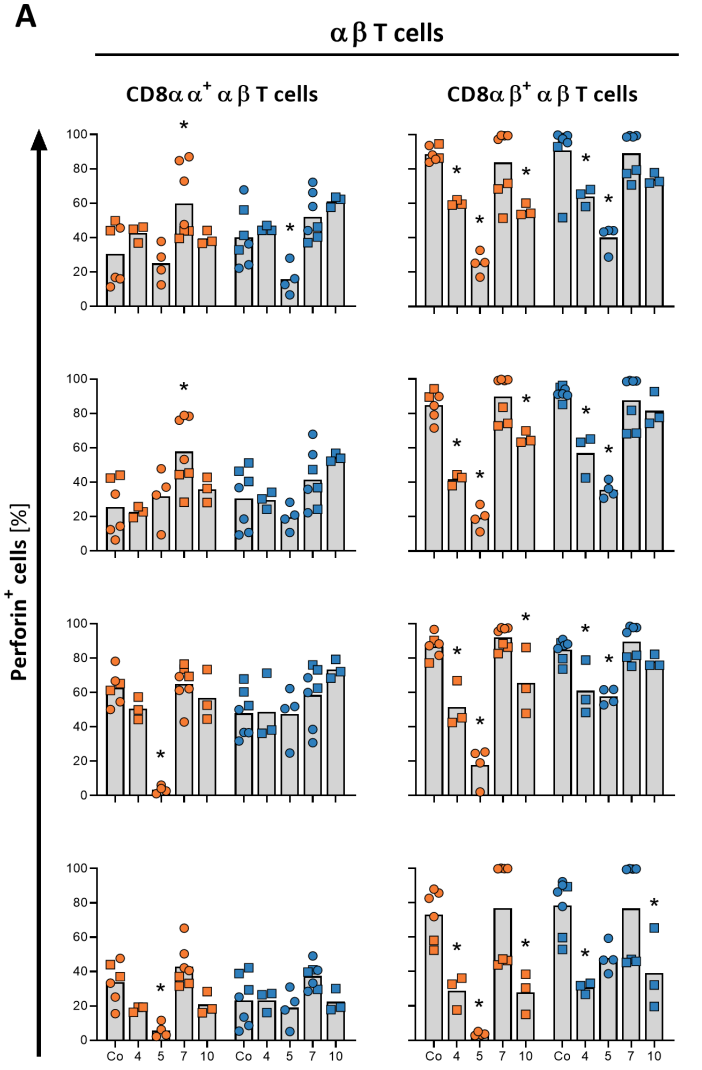

B

$\frac{\gamma \delta \mathrm{T} \text { cells }}{\mathrm{CD2}^{+} / \mathrm{CD}^{+} \alpha^{+} \gamma \delta \mathrm{T} \text { cells }}$
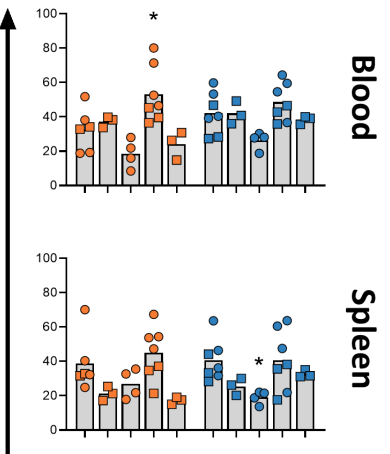

(亡:

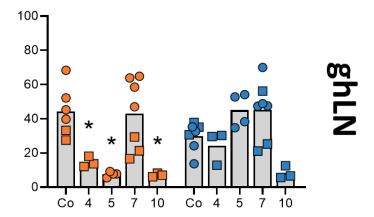


A

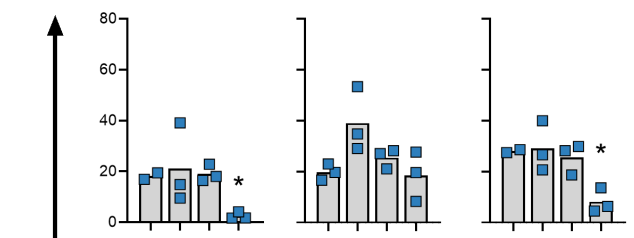

*

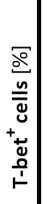
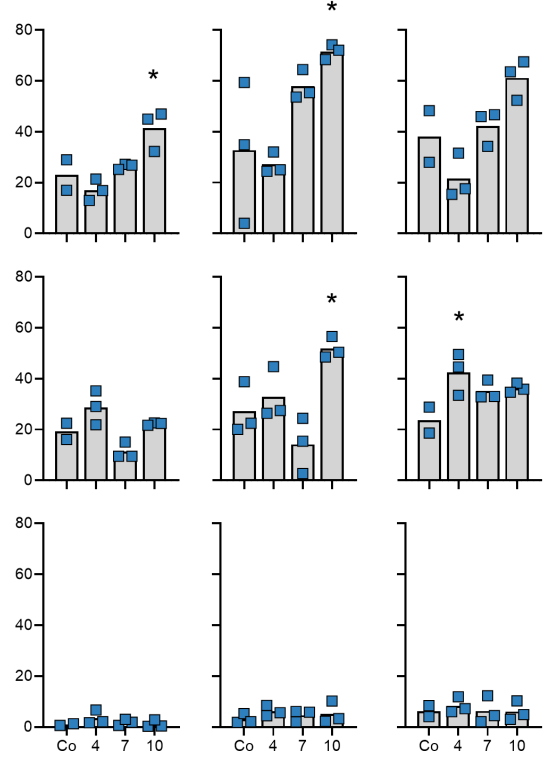

B $\frac{\gamma \delta \text { T cells }}{\mathrm{CD2}^{+} / \mathrm{CD} 8 \alpha^{-} \quad \mathrm{CD}^{+} / \mathrm{CD} 8 \alpha^{+}}$
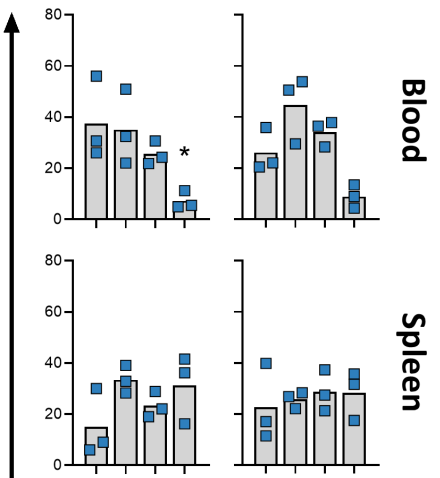

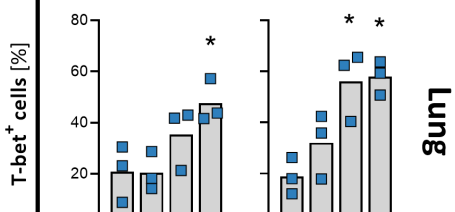
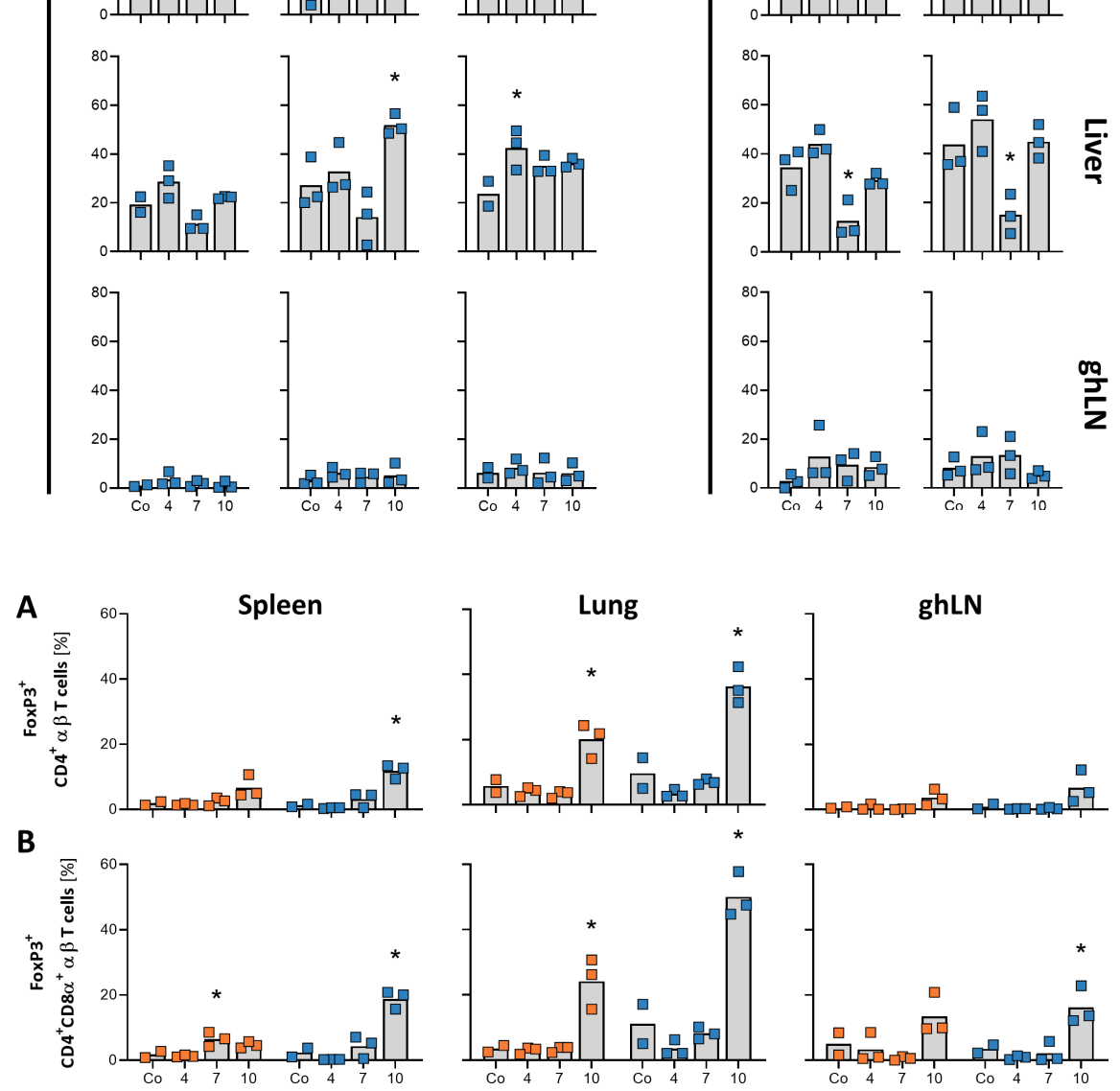

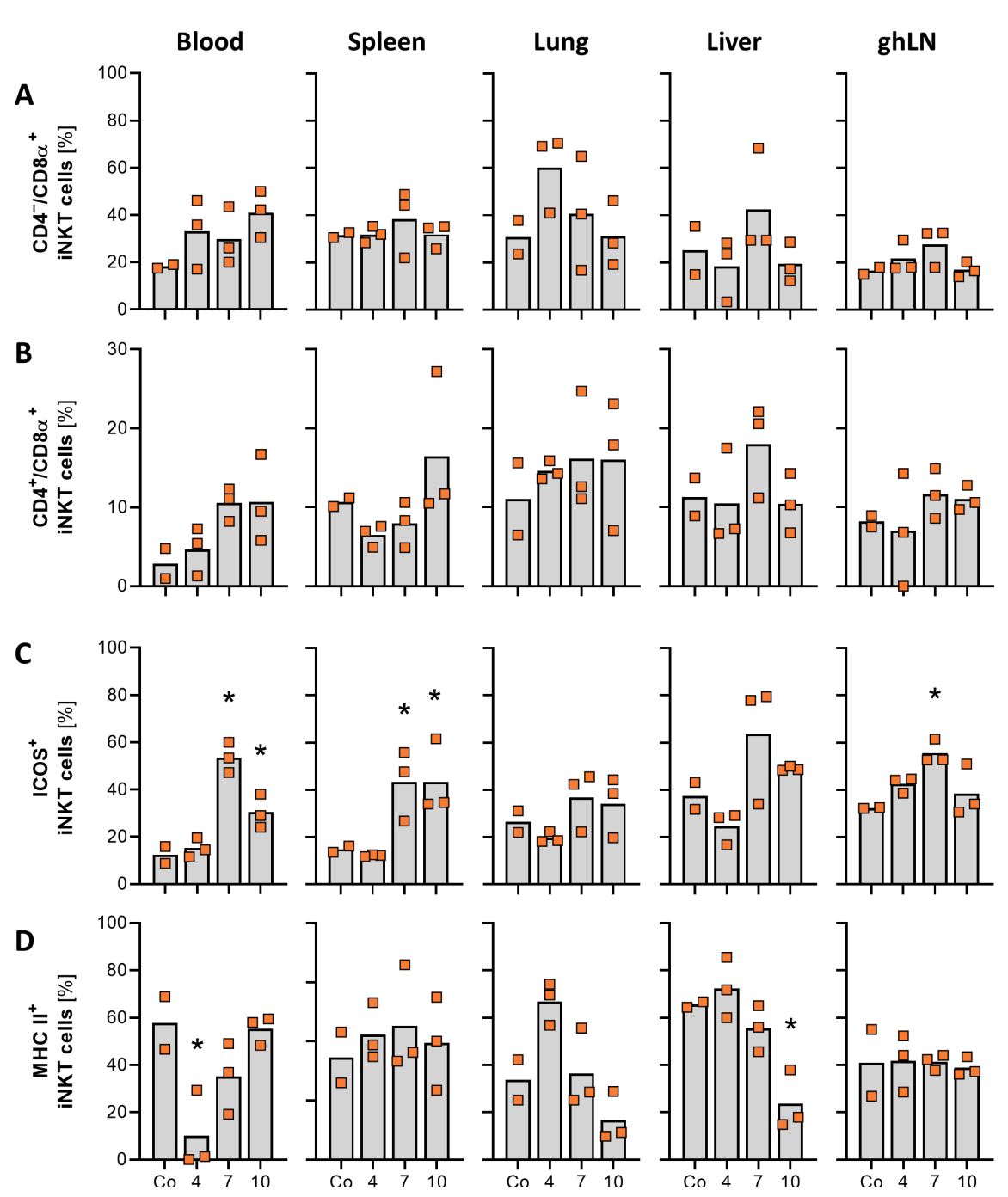